\title{
The MIMO Antenna Array with Mutual Coupling Reduction and Cross-polarization Suppression by Defected Ground Structures
}

\author{
Zhaolin ZHANG ${ }^{1}$, Kun $W E I^{2}$, Jian XIE ${ }^{1}$, Jianying $L I^{1}$, Ling $W A N G{ }^{1}$ \\ ${ }^{1}$ School of Electronics and Information, Northwestern Polytechnical University, 710072 Xi'an, Shannxi, China \\ ${ }^{2}$ School of Electronics Engineering and Computer Science, Peking University, Beijing 100871, China \\ z184@sina.com, mark645276749@163.com, \{xiejian, jianyingli, lingwang\}@nwpu.edu.cn
}

Submitted April 16, 2018 / Accepted October 7, 2018

\begin{abstract}
This paper proposes two types of defected ground structures (DGSs) for achieving the mutual coupling (MC) reduction and the antenna cross-polarization (XP) suppression respectively in a MIMO (multiple input multiple output) wireless communication system. The novel periodic fractal DGS (PFDGS) are presented to reduce the $M C$ between antenna elements. About $20 \mathrm{~dB}$ $M C$ reduction is achieved, which contributes to improve the antenna efficiency and increases the MIMO system channel capacity. However, the method of using DGS or other decoupling structures for MC reduction will degrade the antenna XP level unnecessarily. For solving this problem, another arc-shaped DGS is etched under each patch to suppress the antenna XP level. In this way, the XP level is suppressed from $-10 \mathrm{~dB}$ to $-34.6 \mathrm{~dB}$ in the boresight direction. Moreover, the arc-shaped DGS will not degrade the $M C$ reduction performance.
\end{abstract}

\section{Keywords}

DGS, mutual coupling suppression, fractal structure, cross-polarization, MIMO system

\section{Introduction}

Microstrip antennas are popularly used in civil and military applications for the advantages like inexpensive to manufacture, compact in size, the ability to create high gain arrays in a low-profile. The high antenna isolation is one of the key merits in many applications, especially for the multiple input multiple output (MIMO) wireless communication system. The correlated received signals in different antennas may reduce the system corresponding capacity. Therefore, the MC suppression is very important for the MIMO communication system.

Using the special band-gap structures is one of the efficient ways to increase antenna isolation. The DGS filter- ing characteristic has been used to decouple the microstrip antenna elements [1], [2]. The DGS, which consists of concentric circular rings [3], is developed to reduce the $\mathrm{MC}$ between two microstrip antenna elements. About $5 \mathrm{~dB}$ isolation improvement is revealed by suppressing the surface waves. Two different folded split-ring resonators are proposed in [4], which are etched in the ground plane. More than $30 \mathrm{~dB}$ antenna isolation improvement is obtained. A compact and simple DGS, which consists of two U-shape DGS units, is presented to reduce the MC level [5], in which about $10 \mathrm{~dB} \mathrm{MC}$ suppression is achieved. The DGS structure, which is based on complementary split-ring resonator, is presented to suppress $\mathrm{MC}$ (about $10 \mathrm{~dB}$ ) between microstrip antenna elements [6]. When designing the band-gap DGS filter, the minimal dimensions of the equivalent circuit are desired. The fractal structures help us to generate longer equivalent current line on a small surface [7]. Thus, the dimension of the DGS is suppressed when the fractals are inserted. A Minkowski fractal DGS is used to decrease the $\mathrm{MC}$ in a compact array in [8]. About $7 \mathrm{~dB}$ $\mathrm{MC}$ suppression is realized. The novel fractal structure in [9] achieves more than $35 \mathrm{~dB}$ MC suppression, but it degrades the XP performances. This paper presents the method to keep the XP in a low level when achieving MC suppression. The PFDGS in this paper is completely different with the DGSs in reference [9].

Since the antenna elements isolation is very important for the MIMO systems [10], the MC suppression using DGS in the MIMO wireless communication system has been studied. A T-shape slot impedance transformer is presented to suppress the MC between closely placed PIFAs for MIMO wireless terminals [11]. The MIMO antenna element with high isolation is achieved. The presented PIFA array provides better channel capacity performance. With a DGS in the ground plane, the high isolation (which is below $-25 \mathrm{~dB}$ ) of the printed antenna elements and the good MIMO channel capacity performance are achieved [12]. The high isolation using DGS in a MIMO system is presented in [13]. The isolation of the antenna elements is $28 \mathrm{~dB}$. 
By utilizing the strong MC between two dipoles in [14], the high isolation and the cross-polarization is less than $-34 \mathrm{~dB}$. In [15], the planar arrangement of the Vivaldi elements is preferred over stacked ones for the MC reduction and XP suppression. However, the techniques discussed in reference [14] and [15] are not designed for microstrip antennas. Both the MC reduction and the XP suppression in a microstrip antenna array are achieved in [16] by the parasitic isolator and the circular DGS, respectively. However, the DGS for XP suppression degrades the MC reduction performance.

From the previous research works, the DGS can reduce the MC between microstrip antennas efficiently, but it is also criticized for increasing the antenna XP level unnecessarily. For solving the increased XP level problem, two

\begin{tabular}{|c|c|c|c|c|}
\hline papers & $\begin{array}{c}\text { Decoupling } \\
\text { structure }\end{array}$ & $\begin{array}{c}\text { Maximum MC } \\
\text { reduction }\end{array}$ & $\begin{array}{c}\text { Improved } \\
\text { XP level }\end{array}$ & $\begin{array}{c}\text { Antenna } \\
\text { performance }\end{array}$ \\
\hline$[4]$ & Periodic DGSs & $41 \mathrm{~dB}$ & No & Degradation \\
\hline$[5]$ & U-shaped DGS & $10 \mathrm{~dB}$ & No & Improvement \\
\hline$[9]$ & Fractal DGS & $35 \mathrm{~dB}$ & No & Improvement \\
\hline$[13]$ & $\begin{array}{c}\text { Rectangular } \\
\text { DGS }\end{array}$ & $28 \mathrm{~dB}$ & No & Improvement \\
\hline$[14]$ & $\begin{array}{c}\text { Dielectric post } \\
\text { N. G. }\end{array}$ & Yes & Improvement \\
\hline$[15]$ & $\begin{array}{c}\text { Stacked } \\
\text { arrangement }\end{array}$ & $10 \mathrm{~dB}$ & Yes & Improvement \\
\hline$[16]$ & $\begin{array}{c}\text { Parasitic } \\
\text { isolator }\end{array}$ & $20.9 \mathrm{~dB}$ & Yes & Improvement \\
\hline proposed & $\begin{array}{c}\text { U-shaped and } \\
\text { arc-shaped } \\
\text { DGSs }\end{array}$ & $20 \mathrm{~dB}$ & Yes & Improvement \\
\hline
\end{tabular}

Tab. 1. Comparison of the proposed DGSs and other structures.

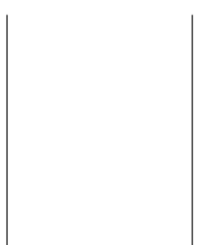

(a)

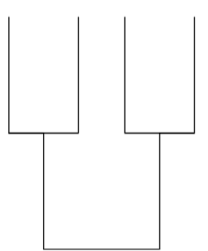

(b)

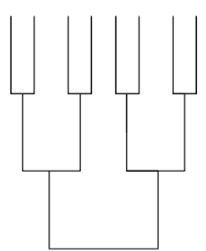

(c)
Fig. 1. The proposed fractal DGS: (a) the zeroth iteration, (b) the first iteration, (c) the second iteration.

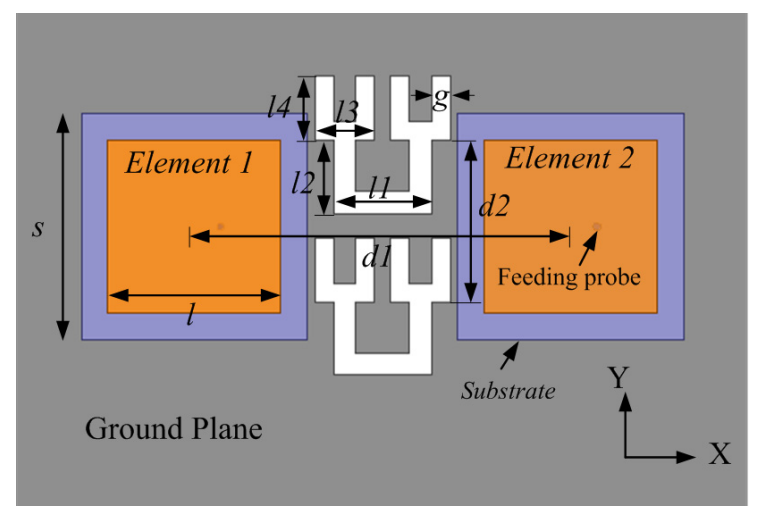

Fig. 2. The geometries of the proposed PFDGS and antenna elements. types of the DGSs are proposed in this paper for realizing the $\mathrm{MC}$ reduction and the XP suppression respectively. Some key results of the proposed DGSs and other decoupling structures are summarized in Tab. 1. The method in this paper achieves MC reduction and XP suppression by the DGSs at the same time. Moreover, the application of the DGS for XP suppression will not degrade the MC reduction performance.

\section{Geometries and Improved Performances}

The proposed fractal structure, as shown in Fig. 1, is evolved from the classic U-shaped structure. Adding two diminutive U-shaped slots at the two endpoints of a basic U-shaped slot turns the zeroth iterative fractal structure into the first iterative fractal structure, as drawn in Fig. 1(b). The third iterative fractal structure is evolved from the the second iterative fractal structure by adding four U-shaped slots at the endpoints. By repeating this process, the high level iterative fractal structures are designed.

The model of the first iterative PFDGS and the microstrip antennas in a MIMO wireless communication system are shown in Fig. 2. Two horizontally polarized microstrip antenna elements are placed on the $\mathrm{x}$-axis. The antennas resonate in the same band with center frequency $3 \mathrm{GHz}$. They have center-to-center distance $d_{1}=50 \mathrm{~mm}$ (equals to 0.5 free-space wavelength). The substrate dielectric constant is 4 with thickness $3.18 \mathrm{~mm}$. The outline dimension of antenna element is $30 \times 30 \times 3.18 \mathrm{~mm}^{3}$, the length of the rectangular patch $l$ is $23 \mathrm{~mm}$. The proposed PFDGS consists of two periodic FDGS etched away from the blank space between microstrip antennas. This designed FDGS unit is evolved from the first iterative fractal structure, as drawn in Fig. 1(b), expanding the first iterative fractal structure with width $g$, and etching the structures from the ground plane. In this way, the proposed PFDGS is designed. The center-to-center distance between the FDGS units is $d_{2}=21.5 \mathrm{~mm}$.

To study the frequency characteristic of the proposed FDGS unit, a microstirp line is settled on a vacuum box above the FDGS unit in ground plane. The parameter studies are carried out to analyze the FDGS unit. There are two factors greatly affecting the FDGS band-gap characteristic, namely the slot length and slot width. The optimized parameters are: $l_{1}=13 \mathrm{~mm}, l_{2}=9.75 \mathrm{~mm}, l_{3}=7.5 \mathrm{~mm}$, $l_{4}=8.5 \mathrm{~mm}$ and $g=2.5 \mathrm{~mm}$.

In Fig. 3 there is the transmission coefficient of the transmission ports with different slot length $l_{4}$ from $7.5 \mathrm{~mm}$ to $9 \mathrm{~mm}$ with step $0.5 \mathrm{~mm}$ when slot width $g=2.5 \mathrm{~mm}$. The increased slot length will decrease the resonant frequency as shown in Fig. 3. The same conclusion can be got when tuning the other FDGS unit length. In Fig. 4, the slot width $g$ is changed from $1.5 \mathrm{~mm}$ to $3.0 \mathrm{~mm}$ when $l_{4}=$ $8.5 \mathrm{~mm}$. As the etched slot width decreases, the operative frequency moves to lower frequency as shown in Fig. 4. In 
general, the increased FDGS dimension will decrease the FDGS resonant frequency.

The comparison of transmission coefficient between the zero and first iterative fractal structures are plotted in

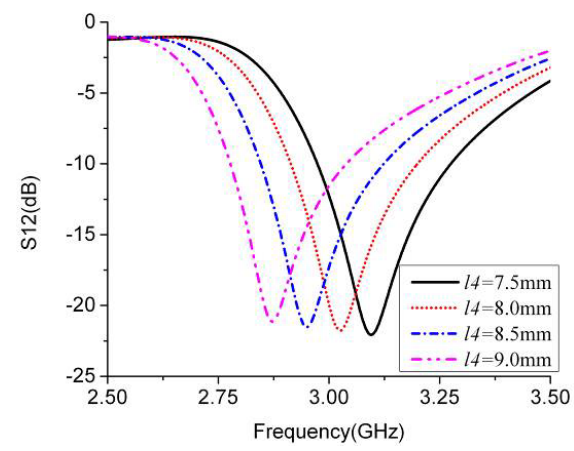

Fig. 3. The transmission coefficients of transmission ports when tuning slot length $l_{4}$.

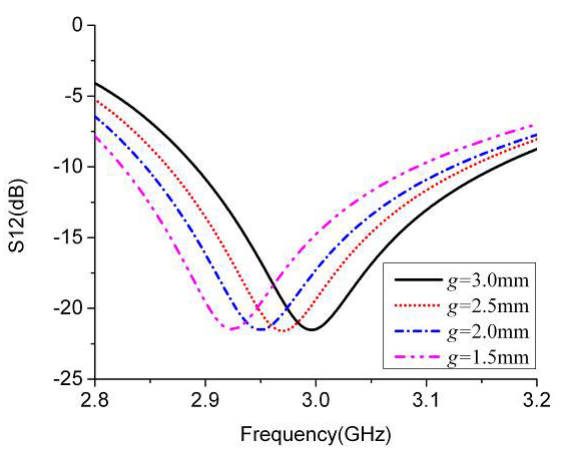

Fig. 4. The transmission coefficients of transmission ports with difference slot width $g$.

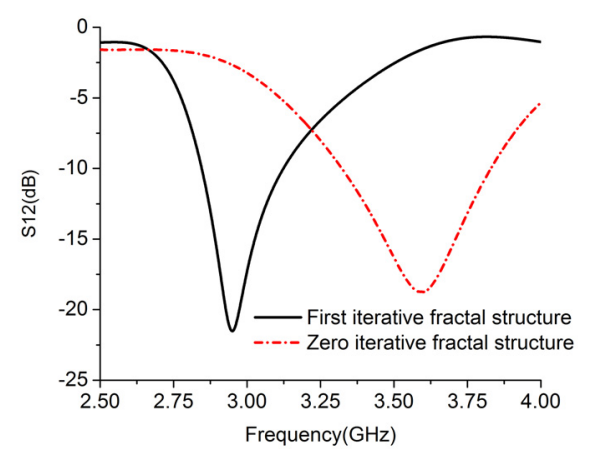

Fig. 5. Transmission coefficient with zero and first iterative fractal structures.

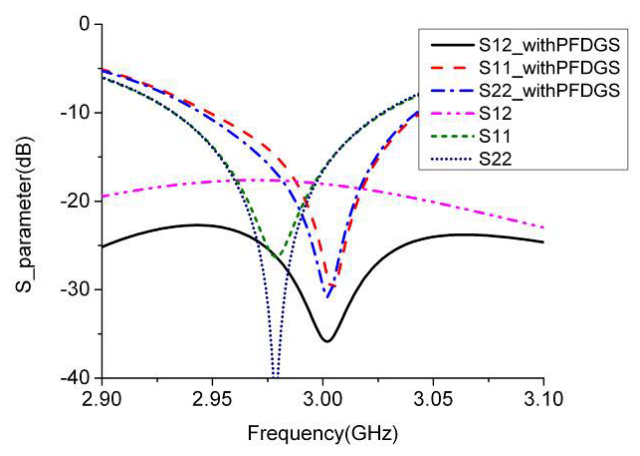

Fig. 6. Mutual coupling suppression with the PFDGS.

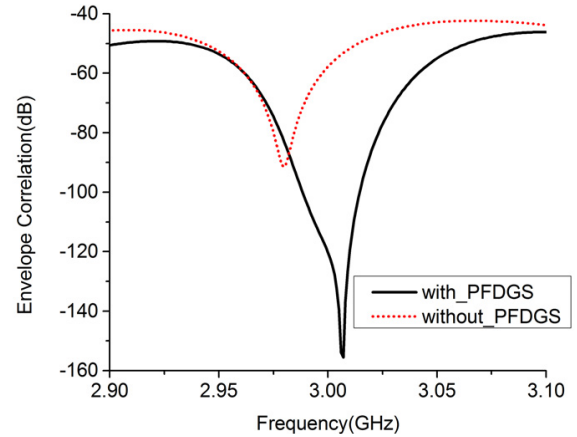

Fig. 7. The array envelope correlation with and without the PFDGS.

Fig. 5. These two structures have the equal outline dimension. When the fractal is inserted, the resonant frequency of the first iterative fractal structure is decreased compared to that of the structure with zero iterative fractal. The first iterative fractal structure is $19.6 \%$ smaller than the zero iterative fractal structure when they have the same resonant frequency.

The optimized parameter values of the first iterative fractal DGS unit are: $l_{1}=13 \mathrm{~mm}, l_{2}=9.75 \mathrm{~mm}, l_{3}=$ $7.5 \mathrm{~mm}, l_{4}=8.5 \mathrm{~mm}$ and $g=2.5 \mathrm{~mm}$. Figure 6 shows the $\mathrm{S}_{11}$ (return loss) and $\mathrm{S}_{12}$ (mutual coupling) of the antennas without and with the presented PFDGS when $d_{1}=50 \mathrm{~mm}$, where $d_{1}$ equals to 0.5 free-space wavelength. The mutual coupling between antenna elements is suppressed from $-16 \mathrm{~dB}$ to $-36 \mathrm{~dB}$ after the PFDGS is etched. About $20 \mathrm{~dB}$ antenna isolation improvement is obtained. Note that the antenna resonant frequency shifts a little to higher frequency band when the PFDGS is inserted. Because the microstrip antenna working frequency can be shifted by adjusting the patch dimension, this method is effective in application.

The envelope correlation of antenna array is a key parameter for the MIMO wireless communication system, because the MC high level may degrade the system corresponding capacity performance. For two elements antenna array in the MIMO communication system, the envelope correlation equation is given by [17]:

$$
\rho_{\mathrm{e}}=\frac{\left|S_{11}{ }^{*} S_{12}+S_{21}{ }^{*} S_{22}\right|^{2}}{\left(1-\left|S_{11}\right|^{2}-\left|S_{21}\right|^{2}\right)\left(1-\left|S_{22}\right|^{2}-\left|S_{12}\right|^{2}\right)} .
$$

Equation (1) is used for calculation of the envelope correlation of the MIMO system in this paper. Figure 7

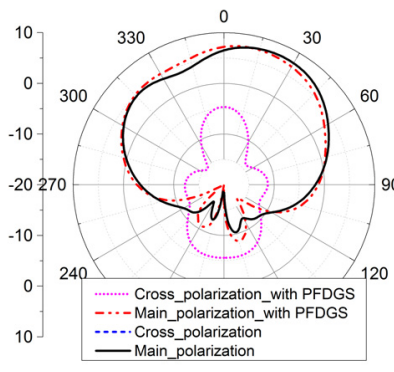

(a)

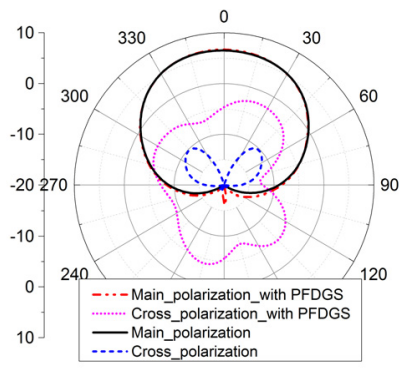

(b)
Fig. 8. Radiation pattern on (a) E-plane and (b) H-plane. 


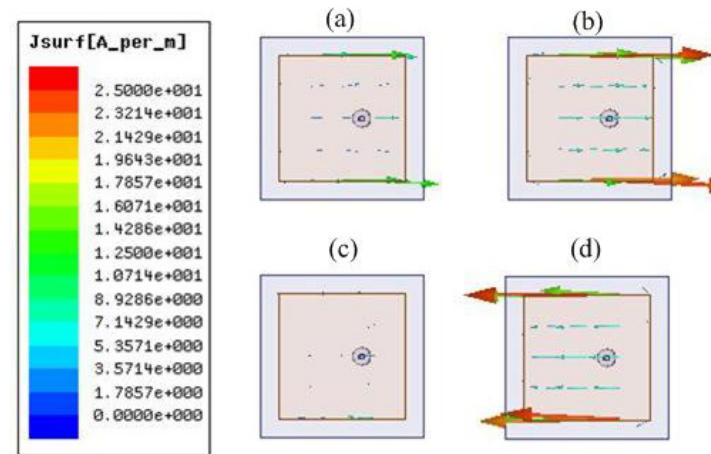

Fig. 9. Surface current vectors of coupled antenna element without PFDGS.

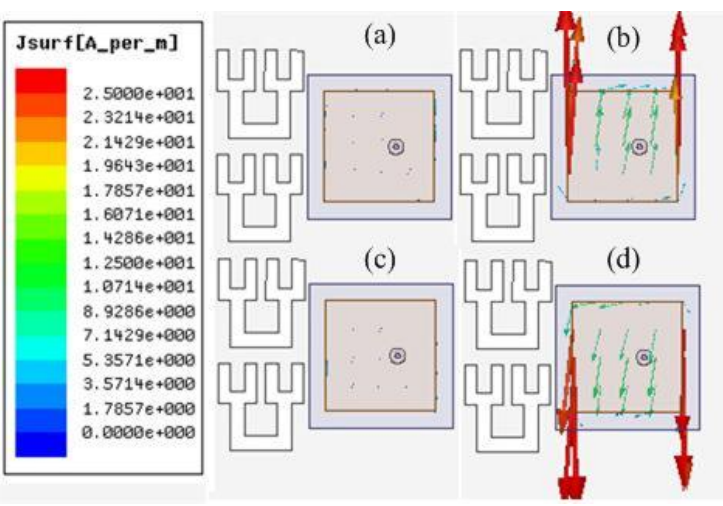

Fig. 10. Surface current vectors of coupled antenna with PFDGS.

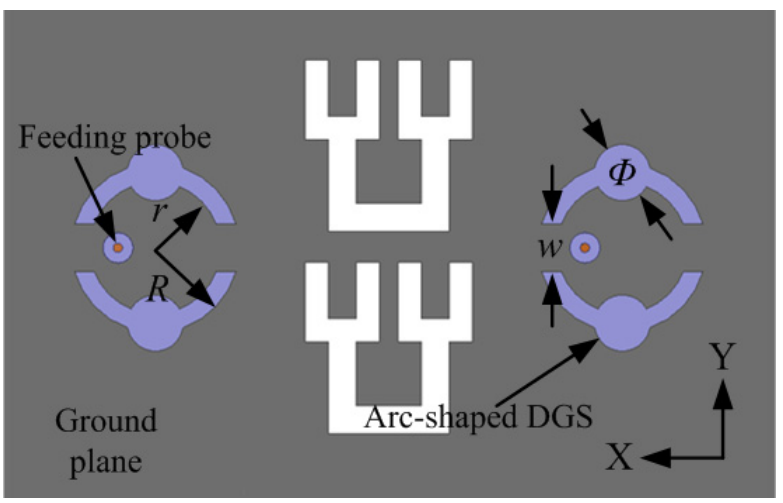

Fig. 11. The geometry of the arc-shaped DGS.

shows the comparisons of the array envelope correlation with and without the proposed PFDGS. Using the designed PFDGS, the envelope correlation value is decreased. The envelope correlation suppression helps to enlarge the MIMO system corresponding capacity. That is to say, high antenna isolation indicates better corresponding capacity performance for the MIMO communication system.

Figure 8 depicts the main polarization and cross-polarization radiation patterns with and without the proposed PFDGS, when only antenna element 2 is excited and the other one is $50 \Omega$ loaded. There is no significant difference between the main radiation patterns in the upper-sphere space on both E-plane and H-plane. However, the antenna XP level with this proposed PFDGS gets greater than that without PFDGS. The antenna XP level on the E-plane without the PFDGS is less than $-40 \mathrm{~dB}$, which cannot be plotted in Fig. 8(a).

The surface current vector graphs of antenna element 2 without PFDGS at time $T=0, \pi / 2, \pi$ and $3 \pi / 2$ are shown in Fig. 9, when antenna element 1 is excited and the other one is $50 \Omega$ loaded. The coupled polarization of element 2 from element 1 is horizontal linear polarization. In addition, element 2 is linear polarized at horizontal direction. Therefore, the received energy of element 2 is a high level, which is radiated by element 1 .

The surface current vector graphs of antenna element 2 with PFDGS at time $T=0, \pi / 2, \pi$ and $3 \pi / 2$ are shown from Fig. 10(a) to Fig. 10(d). Antenna element 1 is excited and the other one is $50 \Omega$ loaded. It can be seen that the coupled polarization of element 2 rotates 90 deg from the horizontal linear polarization to vertical linear polarization using the proposed PFDGS. Thus, the received energy of element 2 , which is radiated by element 1 , is reduced compared to that without PFDGS. In this way, the antenna isolation is improved and XP level is increased. The same conclusion can be got when only antenna element 2 is excited and the other one is $50 \Omega$ loaded.

\section{XP Improvement by Arc-shaped DGS}

The decoupling DGS or other structures may degrade the antenna XP performance when achieving MC reduction, which can be found in many other research works. In order to achieve the antenna XP suppression, the DGSs have been reported in the design [18], [19]. However, only few papers study the DGS for XP suppression in a MC reduction antenna array. For solving the increased XP level problem, another arc-shaped DGS is inserted in the ground plane. The fractal U-shaped DGS and the arc-shaped DGS achieve the $\mathrm{MC}$ reduction and the XP suppression respectively.

As shown in Fig. 11, two pairs of the arc-shaped DGSs are etched in the ground plane, each of them are symmetrically located under the antenna patch. The DGS under the patch works for XP suppression of this element. The arc-shaped DGSs have outside diameter $R$, inside diameter $r$ and angular width $w$. Relative changes in XP values have been studied theoretically by varying the values of $R, r$ and $w$. The optimized parameter values are $R=9 \mathrm{~mm}, r=7 \mathrm{~mm}, \Phi=6 \mathrm{~mm}$ and $w=5 \mathrm{~mm}$. It is found that the angular width $w$ is the key parameter that affects the XP suppression performance.

The $\mathrm{S}_{12}$ of the antenna array versus different values of $w$ are illustrated in Fig. 12. As the angular width $w$ increases, the $\mathrm{MC}$ reduction resonant frequency shifts to the upper region. Note that the minimum values of the $S_{12}$ are all below $-32 \mathrm{~dB}$, which indicates that using the arcshaped DGSs for XP suppression will not degrade the MC reduction performance. 


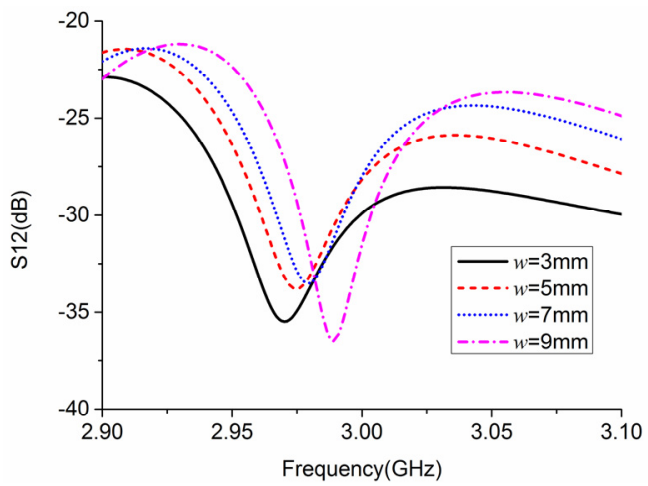

Fig. 12. The $\mathrm{S} 12$ of antenna array when tuning width $w$.

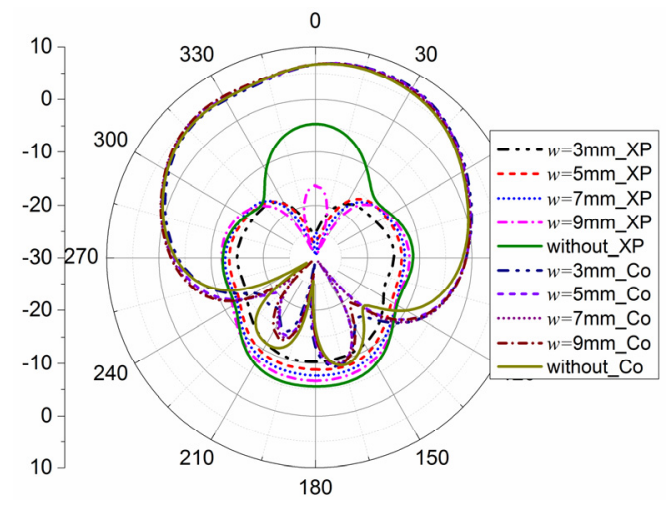

Fig. 13. The radiation patterns on the E-plane when tuning width $w$.

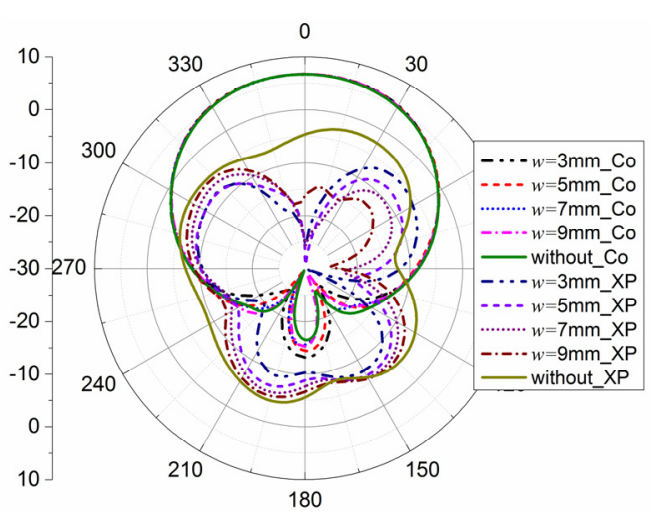

Fig. 14. The radiation patterns on the H-plane when tuning width $w$.

Figure 13 and Figure 14 show the radiation patterns on the E-plane and H-plane versus different values of $w$ when only one antenna element is excited. The XP level is about $-10 \mathrm{~dB}$ in the boresight direction without etching the arc-shaped DGSs. After etching the arc-shaped DGSs in the ground plane, the antenna XP level is obviously suppressed. When the angular width $w$ is optimized to be $5 \mathrm{~mm}$, the antenna XP level is suppressed from $-10 \mathrm{~dB}$ to $-34.6 \mathrm{~dB}$ in the boresight direction. Moreover, there is a slight change with the main polarization of the radiation patterns. It is worthwhile to note that the XP in the other directions (theta from $-45 \mathrm{deg}$ to $45 \mathrm{deg}$ ) are also suppressed with different values.

\section{Simulated and Measured Results}

In order to validate the $\mathrm{MC}$ reduction and the XP suppression performances of the proposed method in this paper, the prototype is fabricated based on the optimized parameters. Figure 15 shows the picture of the testing antennas in a microwave anechoic chamber.

The S parameters of the fabricated antenna elements with and without the proposed two types of the DGSs are plotted in Fig. 16. The S-parameters of the fabricated antennas are measured by using the Agilent E5230A network analyzer. The MC of the antenna array is less than $-35 \mathrm{~dB}$ at the center frequency. About $20 \mathrm{~dB} \mathrm{MC}$ reduction is achieved in both simulation and measurement. Note that the measured $S_{11}$ and $S_{12}$ are shifted to lower frequency. Except for the system and measuring errors, the difference between the measured and simulated results is caused by the machine error of antenna elements and the DGSs, since resonant frequency of the antenna array is sensitive to the patch dimension.

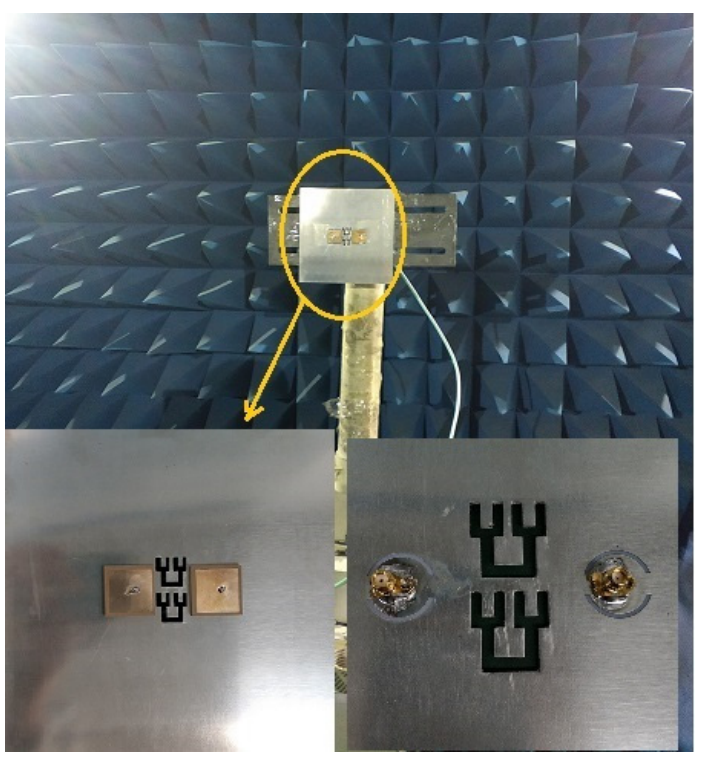

Fig. 15. Picture of the testing antennas in a microwave anechoic chamber.

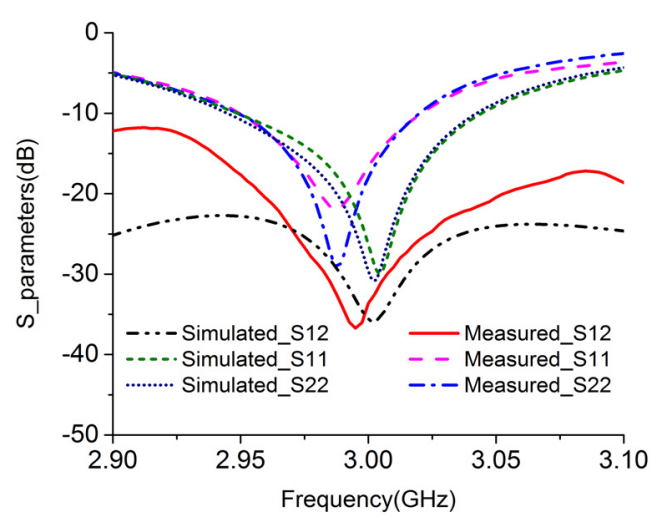

Fig. 16. Simulated and measured $S$ parameters with two types of DGSs. 


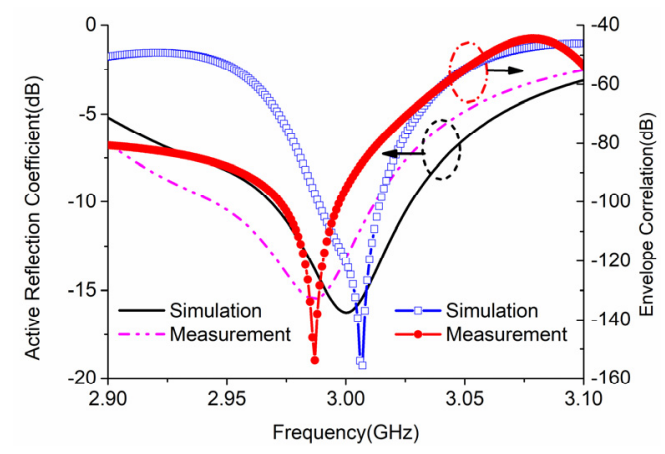

Fig. 17. The simulated and measured active reflection coefficient and envelope correlation of the antenna array.

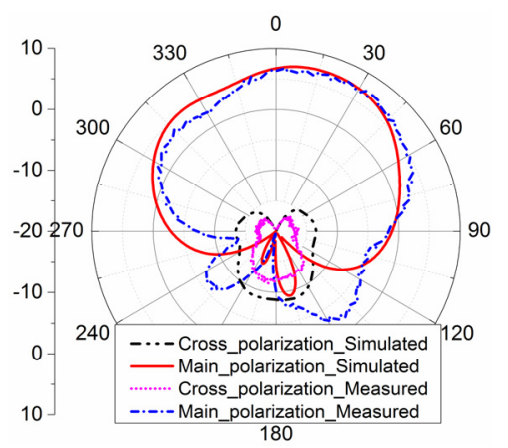

(a)

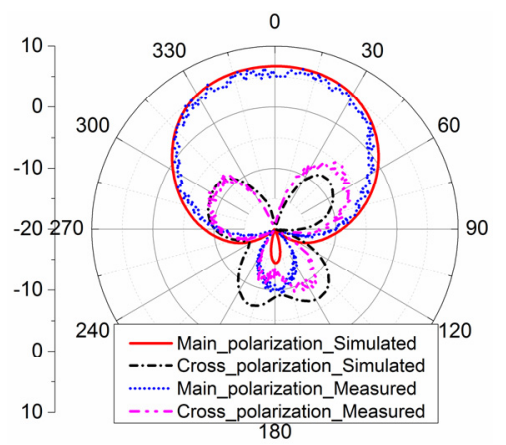

(b)

Fig. 18. Simulated and measured radiation patterns with two types of DGSs on (a) E-plane and (b) H-plane.

Both the simulated and measured active reflection coefficient and envelope correlation of the antenna array are plotted in Fig. 17. The measurements meet well with the simulation. The results show that the MIMO antenna array with the MC reduction has low outgoing power and good corresponding capacity performance.

The simulated and measured main polarization and the cross-polarization radiation patterns of the antenna element 2 are depicted in Fig. 18. These results are measured in a microwave anechoic chamber when only antenna element 2 is excited and the other one is $50 \Omega$ loaded. The measured results agree well with the simulated ones. Antenna efficiency is improved from $76 \%$ to $83 \%$ after etching the proposed DGSs. The radiation patterns in the upper-sphere space have no significant difference between the simulation and measurement. Compared to the radiation patterns of the antennas only with the proposed
PFDGS for the MC reduction, the XP level of the antenna element is suppressed. The measured XP level is $-31 \mathrm{~dB}$ in the boresight direction, which validates the XP suppression by the arc-shaped DGSs. The back-side radiation is increased because of the slots in the ground plane. This problem may be solved by using the proper EBG high-impedance surface structure in the bottom side of the ground plane.

\section{Conclusion}

Two types of DGSs are proposed in this paper for the $\mathrm{MC}$ reduction and XP suppression respectively. A novel PFDGS is studied to realize the MC reduction between antenna elements in a MIMO system. Using the proposed PFDGS, about $20 \mathrm{~dB}$ MC reduction is achieved, which helps to improve the antenna efficiency and to increase the MIMO system channel capacity. However, the application of the DGS or other decoupling structures for MC reduction leads to increase of the antenna XP level. For solving this problem, the arc-shaped DGS is etched in the ground plane under each patch. By doing that, the XP level is suppressed from $-10 \mathrm{~dB}$ to $-34.6 \mathrm{~dB}$ in the boresight direction compared to that of the antennas only with the PFDGS. Both the MC reduction and XP suppression using the DGSs are achieved in this paper.

\section{Acknowledgments}

This study was supported in part by the National Natural Science Foundation of China (No. 61771404, 61601372). This study was especially supported by the China Postdoctoral Science Foundation (No. 2018M631258) and the Postdoctoral Innovative Talent Support Program (No. BX20180003)

\section{References}

[1] WEI, K., LI, J. Y., WANG, L., et al. S-shaped Periodic Defected Ground Structures (PDGS) to reduce microstrip antenna array mutual coupling. Electronics Letters, 2016, vol. 52, no. 15, p. 1288-1290. DOI: 10.1049/el.2016.0667

[2] GUHA, D., BISWAS, S., JOSEPH, T., et al. Defected ground structure to reduce mutual coupling between cylindrical dielectric resonator antennas. Electronics Letters, 2008, vol. 44, no. 14, p. 836-837. DOI: 10.1049/el:20081189

[3] GUHA, D., BISWAS, S., BISWAS, M., et al. Concentric ringshaped defected ground structures for microstrip applications. IEEE Antennas and Wireless Propagation Letters, 2006, vol. 5, no. 1, p. 402-405. DOI: 10.1109/LAWP.2006.880691

[4] HABASHI, A., NOURINIA, J., GHOBADI, C. Mutual coupling reduction between very closely spaced patch antennas using lowprofile folded split-ring resonators (FSRRs). IEEE Antennas and Wireless Propagation Letters, 2011, vol. 10, p. 862-865. DOI: 10.1109/LAWP.2011.2165931 
[5] XIAO, S., TANG, M. C., BAI, Y. Y., et al. Mutual coupling suppression in microstrip array using defected ground structure. IET Microwave, Antennas and Propagation, 2011, vol. 5, no. 12, p. 1488-1494. DOI: 10.1049/iet-map.2010.0154

[6] BAIT-SUWAILAM, M. M., SIDDIQUI, O. F., RAMAHI, O. M. Mutual coupling reduction between microstrip patch antennas using slotted-complementary split-ring resonators. IEEE Antennas and Wireless Propagation Letters, 2010, vol. 9, p. 876-878. DOI: 10.1109/LAWP.2010.2074175

[7] HAMMOODI, A. I., AL-RIZZO, H. M., ISAAC, A. A. Mutual coupling reduction between two monopole antennas using fractal based DGS. In IEEE International Symposium on Antennas and Propagation and USNC/URSI National Radio Science Meeting. Vancouver (Canada), 2015, p. 416-417. DOI: 10.1109/APS.2015.7304594

[8] KAKAOYIANNIS, C. G., CONSTANTINOU, P. Reducing coupling in compact arrays for WSN nodes via pre-fractal defected ground structures. In Proc. of the 39th European Microwave Conference. Roma (Italy), 2009. DOI: 10.23919/EUMC.2009.5295996

[9] WEI, K., LI, J. Y., WANG, L., et al. Mutual coupling reduction by novel fractal defected ground structure band-gap filter. IEEE Transactions on Antennas and Propagation, 2016, vol. 64, no. 10, p. 4328-4335. DOI: 10.1109/TAP.2016.2591058

[10] ZHANG, S., GLAZUNOV, A. A., YING, Z., et al. Reduction of the envelope correlation coefficient with improved total efficiency for mobile LTE MIMO antenna arrays: Mutual scattering mode. IEEE Transactions on Antennas and Propagation, 2013, vol. 61, no. 6, p. 3280-3291. DOI: 10.1109/TAP.2013.2248071

[11] ZHANG, S., LAU, B. K., TAN, Y., et al. Mutual coupling reduction of two PIFAs with a T-shape slot impedance transformer for MIMO mobile terminals. IEEE Transactions on Antennas and Propagation, 2012, vol. 60, no. 3, p. 1521-1530. DOI: 10.1109/TAP.2011.2180329

[12] LI, H., XIONG, J., HE, S. A compact planar MIMO antenna system of four elements with similar radiation characteristics and isolation structure. IEEE Antennas and Wireless Propagation Letters, 2009, vol. 8, p. 1107-1110. DOI: 10.1109/LAWP.2009.2034110.

[13] IBRAHIM, A. A., ABDALLA, M. A., ABDEL-RAHMAN, A. B. et al. Compact MIMO antenna with optimized mutual coupling reduction using DGS. International Journal of Microwave and Wireless Technologies, 2014, vol. 6, no. 2, p. 173-180. DOI: $10.1017 / \mathrm{S} 1759078713001013$

[14] BAO, Z. D., ZONG, X. Z., NIE, Z. P., et al. Design and discussion of a broadband cross-dipole with high isolation and low crosspolarisation utilising strong mutual coupling. IET Microwave, Antennas and Propagation, 2014, vol. 8, no. 5, p. 315-322. DOI: 10.1049/iet-map.2013.0440

[15] ZHANG, C., KUHN, M., MAHFOUZ, M., et al. Planar antipodal Vivaldi antenna array configuration for low cross-polarization and reduced mutual coupling performance. In IEEE Antennas and Propagation Society International Symposium. Honolulu (HI USA), 2007, p. 725-728. DOI: 10.1109/APS.2007.4395596

[16] CHENG, Y,-F., DING, X., SHAO, W., et al. Reduction of mutual coupling between patch antennas using a polarization-conversion isolator. IEEE Antennas and Wireless Propagation Letters, 2017, vol. 16, p. 1257-1260. DOI: 10.1109/LAWP.2016.2631621

[17] BLANCH, S., ROMEU, J., CORDELLA, I. Exact representation of antenna system diversity performance from input parameter description. Electronics Letters, 2003, vol. 39, no. 9, p. 705-707. DOI: $10.1049 / \mathrm{el}: 20030495$

[18] KUMAR, C., GUHA, D. Nature of cross-polarized radiations from probe-fed circular microstrip antennas and their suppression using different geometries of defected ground structure (DGS). IEEE Transactions on Antennas and Propagation, 2012, vol. 60, no. 1, p. 92-101. DOI: 10.1109/TAP.2011.2167921
[19] GUHA, D., KUMAR, C., PAL, S. Improved cross-polarization characteristics of circular microstrip antenna employing arc-shaped defected ground structure (DGS). IEEE Antennas and Wireless Propagation Letters, 2010, vol. 8, p. 1367-1369. DOI: 10.1109/LAWP.2009.2039462

\section{About the Authors ...}

Zhao-Lin ZHANG is the assistant professor in the Northwestern Polytechnical University (NPU). His current research interests include wideband communications, cognitive radio, adaptive anti-jamming for satellite communications and satellite navigation.

Kun WEI received the degrees of M.Sc and Ph.D in Electronic Engineering in the NPU in 2014 and 2017, respectively. Currently he is working as a post-doctor researcher in the School of Electronics Engineering and Computer Science, Peking University. His recent research interests include microstrip antennas, satellite communication antenna, circularly-polarized antenna, periodic structure, and metamaterial.

Jian XIE received the M.Sc. and Ph.D. degree from the School of Electronic Engineering, Xidian University in 2012 and 2015, respectively. He is now an associate professor with the NPU. His research interests include antenna array processing and radar signal processing.

Jian-Ying LI received the degrees of B.Sc. in Mathematics, and M.Eng.Sc. and Ph.D. both in Electromagnetic Field and Microwave Technology from Henan Normal University, Xinxiang, China, in 1986, and Xidian University, Xi'an, China, in 1992 and 1999, respectively. From 1992 to 1996, he worked at Xi'an Electronic Engineering Research Institute, Xi'an, China as a Research Engineer. From 1999 to 2004, he is first with the Dept. of Electrical and Computer Engineering at the National University of Singapore (NUS) where he is a Postdoctoral Research Fellow and then with High Performance Computation for Engineered Systems Programme at the Singapore-MIT Alliance where he is a Research Fellow. From 2005 to 2010, he is with the Temasek Laboratories, NUS, where he is a research scientist. Since 2011 he has been with the School of Electronic and Information, NPU. His current research interests include the fast algorithms and their applications to radar cross sections, analysis and design of phased arrays, waveguide slot antennas, and microstrip antennas, and EM Periodic Structures.

Ling WANG received the degrees of B.Sc. M.Sc. and Ph.D. from Xidian University, Xi'an, China, in 1999, 2002 and 2004, respectively, all in Electronic Engineering. From 2004 to 2007, he worked at Siemens and Nokia Siemens Networks. Since 2007, he has been with the School of Electronic and Information, NPU and was promoted to Professor in 2012. His current research interests include array processing and smart antennas, wideband communications, cognitive radio, adaptive anti-jamming for satellite communications, satellite navigation, and date link systems. 